\title{
The psychological impact of COVID-19 on ear, nose and throat (ENT) specialists
}

\author{
Thomas J. Crotty ${ }^{1}$ (I) $\cdot$ Mel Corbett ${ }^{1}$ - Stephen Garry ${ }^{2} \cdot$ Matthew G. Davey $^{2} \cdot$ Joseph P. Hughes $^{3} \cdot$ Ivan J. Keogh $^{2}$. \\ Nash P. Patil ${ }^{1} \cdot$ Eva Doherty ${ }^{4}$
}

Received: 31 January 2021 / Accepted: 19 February 2021 / Published online: 25 February 2021

(c) Royal Academy of Medicine in Ireland 2021

\begin{abstract}
Introduction Ear, nose and throat (ENT) specialists are a high-risk group for COVID-19. Although the implications of SARS-CoV-2 infection on physical health are well-documented, the psychological impact is frequently overlooked.

Aims The aim of this study was to gauge the psychological impact of COVID-19 on ENT specialists in Ireland.

Methods A national, cross-sectional, web-based survey was distributed to ENT specialists during the peak of the COVID19 pandemic ( $21^{\text {st }}$ May 2020-21 $1^{\text {st }}$ June 2020). The questionnaire collected sociodemographic and COVID-19 related data. The GAD-7 was utilized to measure symptoms of clinically significant anxiety disorder.

Results Thirty-eight ENT specialists (M/F:24/12, median age, 38.7 years (23-60 years)) completed the survey. About 34\% $(n=13)$ of participants screened positive for an anxiety disorder, of which $2(5 \%)$ had moderate symptoms. The majority of participants $(n=32,84 \%)$ felt ENT specialists had increased exposure to SARS-CoV-2 compared with other medical specialties. Additionally, $32 \%(n=12)$ felt incapable of protecting themselves from infection. An encouraging proportion of ENT specialists $(n=22,58 \%)$ were aware of psychological support available from national and institutional organizations. Conclusions The long-term psychological sequelae of COVID-19 will be felt as the physical burden eases. As we adjust to new normalities, ENT surgeons must be conscientious of the mental health issues that arise from the working environment. Sources of anxiety emanated from a lack of control over infection risk, increased vulnerability to COVID-19 relative to other specialties and the implications this has for ENT specialists and their families. Future interventions must focus on increasing awareness of the available psychological support services for our healthcare workers.
\end{abstract}

Keywords Anxiety $\cdot$ COVID-19 $\cdot$ ENT $\cdot$ Mental health $\cdot$ Otolaryngology

\section{Introduction}

Coronavirus disease 2019 (COVID-19) originated in Wuhan, Hubei Province, China, in November 2019. The virus rapidly disseminated to more than 200 countries, infecting 19

Thomas J. Crotty

tcrotty92@gmail.com

1 Department of Otolaryngology/Head and Neck Surgery, Sligo University Hospital, The Mall, Sligo, Ireland

2 Department of Otolaryngology/Head and Neck Surgery, University Hospital Galway, Newcastle Road, Galway, Ireland

3 Department of Otolaryngology/Head and Neck Surgery, University Hospital Limerick, Dooradoyle, Limerick, Ireland

4 Department of Human Factors and Patient Safety, Royal College of Surgeons, St. Stephen's Green, Dublin, Ireland million people and causing 726,000 deaths worldwide [1]. Ireland declared a national state of emergency on the 19 March 2020, nearly 3 weeks after the first case was diagnosed. Public health legislation initially focused on reducing person-to-person transmission by recommending social distancing and self-isolation, before mandating a nationwide lockdown on 27 March 2020. Meanwhile, health organisations cancelled elective procedures in order to maximise the resources and facilities available to patients with COVID19 requiring hospitalisation and critical care support [2]. Despite efforts to prepare for the surge of patients with COVID-19, the expeditious rise in cases, rapid consumption of personal protective equipment (PPE) and prospect of being redistributed to an unfamiliar speciality provoked fear and anxiety amongst healthcare workers (HCW).

$\mathrm{HCW}$ are a well-established high-risk group for contracting COVID-19 with over 32\% of all cases diagnosed in 
Ireland falling into this category [3]. Although the physical health complications arising from COVID-19 infection has received extensive coverage, the psychological impact that the COVID-19 pandemic incurs on healthcare workers has not garnered much attention. One year following the SARS outbreak in China, researchers found significantly higher levels of depression, anxiety and post-traumatic stress disorder in HCW when compared with non-HCW in highly affected regions [4]. A cross-sectional study of doctors working in Ireland before the COVID-19 pandemic found that $50 \%$ reported being emotionally exhausted and overwhelmed by their work, while $30 \%$ were experiencing symptoms of burnout, a comparably higher figure than in the UK, USA, or Australia [5]. Considering the high baseline level of emotional exhaustion amongst our nation's doctors, the superimposed effects of the COVID-19 outbreak may result in significant, long-term deteriorations in mental health.

Ear, nose and throat (ENT) specialists have been particularly affected by the COVID-19 pandemic by virtue of the wide array of upper aerodigestive tract examinations and interventions they perform. Early experiences from Wuhan, China, reported that ENT surgeons were at increased risk of infection due to the high viral load of SARS-CoV-2 in the upper respiratory tract and nasopharynx [6]. The Irish Head and Neck Society (IHNS) guidelines initially recommended the cancellation of walk-in ENT clinics and routine operations due to the substantial risk of viral exposure through aerosolized secretions or aerosol generating procedures (AGPs), such as tracheostomy and laryngoscopy [7]. Despite the cancellation of many upper aerodigestive tract procedures, emergency, and time-sensitive, necessary oncological procedures were still being carried out. The increased vulnerability associated with caring for patients with head and neck pathology may be undermining the mental well-being of ENT specialists, placing them at increased risk for clinically significant mental health problems, including generalized anxiety disorder and post-traumatic stress disorder. Our aim is to gauge the psychological impact of the COVID-19 pandemic on ENT surgeons in Ireland.

\section{Methods}

We conducted a national, cross-sectional, descriptive study of ENT specialist's experience from 21 May to 21 June 2020. The study was approved by the National Research Ethics Committee for COVID research, and informed consent was obtained prior to data collection. Invitations to participate in this study were distributed to the email addresses of registered members of the Irish Institute of Otorhinolaryngology/Head and Neck Surgery (IIOHNS). Our target cohort included doctors of all grades working within an ENT service in Ireland during the COVID-19 pandemic.
Participants involved in the development of the questionnaire were excluded from the study.

The survey instrument was developed using a pilot study performed on a small, representative sample of participants, who were ultimately excluded from participating in the main study. Respondents received an email with a direct link to the structured questionnaire via an online survey platform (SurveyMonkey Inc). Sociodemographic and COVID-19related data was obtained. Sociodemographic data included participant's age, gender, hospital network, professional title and living arrangements. COVID-19-specific data included compliance with social distancing, previous COVID-19 testing, direct contact with COVID-19 patients, accessibility to PPE, alterations to normal working hours, and perceived risk of COVID-19 transmission.

ENT specialists' anxiety was measured using Spitzer's 7-item General Anxiety Disorder scale (GAD-7) [8]. The GAD-7 contains 7-items which assess the presence of clinically significant anxiety disorder. Respondents were asked how frequently they experience anxiety-related symptoms on a 1-4 point scale, with 0 being "not at all" and 4 being "every day." The final result is between 0 and 28 with cutoffs at 5,10 , and 15 , interpreted as mild, moderate, and severe levels of anxiety, respectively.

\section{Results}

\section{Sociodemographic data}

Thirty-eight participants returned the survey, yielding a $40.8 \%$ response rate. Table 1 depicts the participants' sociodemographic characteristics. The mean age of the respondents was 38.7 years old (range, 23-60 years). There was a 2:1 male-to-female preponderance (male, $n=24$; female, $n=12$ ), with 2 participants not disclosing their gender. Fifteen consultants, 14 registrars, 7 senior house officers and 2 interns answered the survey. A total of 21 ENT specialists (64\%) were living with other HCW during the COVID-19 pandemic.

\section{COVID-19-related data}

Table 2 illustrates COVID-19 related data for ENT specialists in Ireland. Twenty-five participants $(66 \%)$ reported that they were usually able to comply with social distancing at work. Out of the 25 participants, only 12 $(32 \%)$ rated their compliance with social distancing as "good." Nine participants (24\%) reported usually being able to comply with social distancing at home, and 13 (34\%) participants were always able to comply with social distancing at home. Only two (5\%) respondents reported having direct patient contact less frequently than once a 
Table 1 Baseline characteristics of participants

\begin{tabular}{|c|c|}
\hline Sociodemographic variables & $\begin{array}{l}\text { ENT specialists } \\
n=38\end{array}$ \\
\hline Age (median (range)) & 38.71 years $(23-60)$ \\
\hline \multicolumn{2}{|l|}{ Gender $(n(\%))$} \\
\hline Male & $24(63 \%)$ \\
\hline Female & $12(32 \%)$ \\
\hline \multicolumn{2}{|l|}{ Hospital group $(n(\%))$} \\
\hline Saolta University & $12(32 \%)$ \\
\hline Ireland East & $7(18 \%)$ \\
\hline RCSI & $6(16 \%)$ \\
\hline South/South-West & $3(8 \%)$ \\
\hline Dublin Midlands & $4(11 \%)$ \\
\hline University Limerick & $2(5 \%)$ \\
\hline Other & $4(11 \%)$ \\
\hline \multicolumn{2}{|l|}{ Professional title $(n(\%))$} \\
\hline Consultant & $15(39 \%)$ \\
\hline Registrar & $14(37 \%)$ \\
\hline Senior House Officer & $7(18 \%)$ \\
\hline Intern & $2(5 \%)$ \\
\hline \multicolumn{2}{|c|}{ Shared accommodation with HCW ( $n(\%))$} \\
\hline Yes & $21(55 \%)$ \\
\hline No & $17(45 \%)$ \\
\hline \multicolumn{2}{|c|}{ Underlying medical comorbidity $(n(\%))$} \\
\hline Yes & 0 \\
\hline No & $33(87 \%)$ \\
\hline Unsure & $5(13 \%)$ \\
\hline
\end{tabular}

week, whereas 23 (61\%) respondents had direct patient contact every day. In spite of this, a minority of respondents $(n=14,37 \%)$ had been tested for COVID-19 at the time of this survey, of which, $4(11 \%)$ were positive. Fifty percent of ENT specialists $(n=19)$ in this study felt the majority of their patient contact took place in the outpatients department, followed by inpatient wards $(n=9$, $24 \%)$, the emergency department $(n=8,21 \%)$ and the operating theatre $(n=2,5 \%)$.

Overall, 89\% ( $n=34)$ of ENT participants had experienced a reduction in team members from a combination of positive cases, self-isolation and redeployment of staff. A minority of participants $(n=7,18 \%)$ were redistributed to critical care $(n=2)$, emergency medicine $(n=2)$, internal medicine $(n=2)$ and general surgery $(n=1)$. With regard to the provision of PPE, $58 \%(n=22)$ of ENT specialists reported having PPE available for all direct patient encounters, while 40\% $(n=15)$ had PPE available for most of their encounters. Only 1 respondent found the provision of PPE unsuitable for managing patients. Twenty-seven participants (71\%) were satisfied with their PPE training.
Table 2 Participants' COVID-19-related data

\begin{tabular}{ll}
\hline COVID-19 related variables & ENT specialists \\
& $n=38$
\end{tabular}

Ability to comply with social distancing at work

$(n(\%))$

$\begin{array}{ll}\text { Always } & 0 \\ \text { Usually } & 25(66 \%) \\ \text { Sometimes } & 9(24 \%) \\ \text { Rarely } & 4(11 \%) \\ \text { Never } & 3(8 \%)\end{array}$

Ability to comply with social distancing at home $(n(\%))$

$\begin{array}{ll}\text { Always } & 13(34 \%) \\ \text { Usually } & 9(24 \%) \\ \text { Sometimes } & 8(21 \%) \\ \text { Rarely } & 5(13 \%) \\ \text { Never } & 3(8 \%)\end{array}$

Actual compliance with social distancing at work $(n(\%))$

Good $12(32 \%)$

Fair $18(47 \%)$

Poor $\quad 7(18 \%)$

Very poor $1(3 \%)$

Tested for COVID-19 ( $n(\%))$

Yes $14(37 \%)$

No $24(63 \%)$

Team reduced from positive cases, self-isolation, redeployment $(n(\%))$

$\begin{array}{ll}\text { Yes } & 34(89 \%) \\ \text { No } & 4(11 \%)\end{array}$

Frequency of direct patient contact $(n(\%))$

Every day $\quad 23(61 \%)$

Few times a week $13(34 \%)$

Once a week $1(3 \%)$

Few times a month $\quad 1(3 \%)$

Where does majority of patient contact take place $(n(\%))$

Inpatient wards $9(24 \%)$

Outpatients department $19(50 \%)$

Operating theatre $2(5 \%)$

Emergency department $8(21 \%)$

Access to PPE during direct patient contact $(n(\%))$

$\begin{array}{ll}\text { All of the time } & 22(58 \%) \\ \text { Most of the time } & 15(39 \%) \\ \text { Sometimes } & 1(3 \%) \\ \text { Never } & 0\end{array}$

PPE suitable to manage patients $(n(\%))$

Yes $\quad 37(97 \%)$

No $1(3 \%)$

Satisfaction with PPE training ( $n(\%))$

Very satisfied $\quad 11(29 \%)$

Satisfied $16(42 \%)$

Neither $7(18 \%)$ 
Table 2 (continued)

\begin{tabular}{ll}
\hline COVID-19 related variables & $\begin{array}{l}\text { ENT specialists } \\
n=38\end{array}$ \\
\hline $\begin{array}{l}\text { Dissatisfied } \\
\text { Redeployed to work outside usual specialty }(n(\%))\end{array}$ & $4(11 \%)$ \\
Yes & $7(18 \%)$ \\
No & $31(82 \%)$ \\
\hline
\end{tabular}

\section{Anxiety amongst ENT specialists}

We utilized the GAD-7 scale to assess the severity of anxiety in our participants (Table 3). The overall prevalence of anxiety disorder was $34 \%(n=13)$, with a median GAD-7 score of 3.2 (range 0-10). Eleven (29\%) ENT specialists were classified as experiencing mild anxiety disorder, and $2(5 \%)$ were classified as experiencing moderate anxiety disorder. No participants had symptoms consistent with
Table 3 Participants' anxiety and other mental health related data

\begin{tabular}{ll}
\hline Psychological variables & $\begin{array}{l}\text { ENT specialists } \\
n=38\end{array}$ \\
\hline
\end{tabular}

Anxiety (GAD-7)

No anxiety $(<5)$

$25(66 \%)$

Mild anxiety (5-9)

$11(29 \%)$

Moderate anxiety (10-14)

Severe anxiety $(>15)$

$2(5 \%)$

0

More or less exposed than other surgical specialties $(n(\%))$

More exposure

Same level

$4(11 \%)$

Less exposure

$2(5 \%)$

Likelihood of contracting COVID-19 ( $n(\%))$

Very likely

$6(16 \%)$

Likely

$11(29 \%)$

Neither likely or unlikely

$12(32 \%)$

Unlikely

$8(21 \%)$

Very unlikely

$1(3 \%)$

Capability of protecting yourself from COVID-19 ( $n(\%))$

Very capable

$1(1 \%)$

Capable

$19(50 \%)$

Neither capable nor incapable

$6(16 \%)$

Incapable

$12(32 \%)$

Very incapable

0

Location with most exposure to COVID-19 ( $n(\%))$

Emergency department

$16(42 \%)$

Operating theatre

$5(13 \%)$

Outpatients department

$12(32 \%)$

Community

$3(8 \%)$

Other

$2(5 \%)$

Procedure with most exposure $(n(\%))$

Tracheostomy formation

$24(63 \%)$

Epistaxis management

$9(24 \%)$

Flexible nasendoscopy

$5(13 \%)$

Quinsy drainage

0

Ear microsuction

0

Awareness of supports available during COVID-19 pandemic $(n(\%))$

Yes

$16(42 \%)$ 
severe anxiety disorder. Thirty-two (84\%) participants felt they were at higher risk of being exposed to SARS-CoV-2 than other medical specialties. Forty-five percent $(n=17)$ of ENT specialists felt they were likely to contract COVID19 , and $32 \%(n=12)$ felt incapable of protecting themselves from becoming infected. Most respondents $(n=16,45 \%)$ perceived themselves as most vulnerable while in the emergency department. With regard to procedures, the majority of respondents $(n=24,63 \%)$ felt tracheostomy was the highest-risk procedure, while $24 \%(n=9)$ and $13 \%(n=5)$ felt managing epistaxis and conducting flexible nasendoscopy were most risky, respectively. Microsuctioning of the external auditory canal was generally perceived as the safest procedure $(n=30,79 \%)$. Most ENT specialists $(n=22,58 \%)$ were aware of support services available during the COVID19 pandemic. Of these 22, 12 (32\%) recognized the Royal College of Surgeons Ireland (RCSI) and the Health Service Executive (HSE) as a potential source of support.

\section{Discussion}

This is the first study examining the psychological impact of the COVID-19 pandemic on ENT specialists. The prevalence of anxiety among the participants in this study was high, affecting $34 \%$ of patients. Reported causative factors for anxiety included the perception of increased vulnerability when compared with other specialties and diminished capacity to protect oneself from infection. A wide array of potential AGPs are performed by ENT specialists, of which respondents felt tracheostomy insertion, epistaxis management and flexible nasendoscopy posed the highest risk of viral transmission. A reasonable proportion of participants were aware of sources for psychological support; however, increased resources towards supporting the emotional and physical needs of high-risk specialties are needed.

Over one-third (34\%) of ENT specialists in our study screened positive for an anxiety disorder using the GAD-7 scale. While the majority of participants had symptoms consistent with mild anxiety, two participants (5\%) met the criteria for moderate anxiety. As there are no studies in the literature examining the rate of anxiety in ENT specialists prior to the COVID-19 pandemic, it is difficult to directly compare the level of anxiety before and after the start of the pandemic. One of the few studies investigating the rate of anxiety in doctors before the COVID-19 outbreak screened the mental health of 227 oncologists, a medical profession traditionally associated with experiencing high levels of emotional distress [9]. In this study, the researchers found the rate of anxiety to be $19.4 \%$, considerably lower than the rate in our study. Comparing our results with a recent study investigating the rate of anxiety in a group of $449 \mathrm{HCW}$ working in ENT departments throughout Hubei province during COVID-19, we reported a 5\% higher rate of anxiety overall [10]. Additionally, the largest meta-analysis utilizing the GAD-7 screening tool to examine the prevalence of anxiety in HCW during the COVID-19 pandemic reported similar rates of anxiety to the ENT specialists in our cohort [11]. Considering the significant psychological and physical strain on HCW, we postulate that our findings, consistent with recent studies, reflect an elevated rate of anxiety amongst ENT specialists in Ireland. However, the long-term consequences of this are unclear. Chronic psychological stress can be associated with dysregulation of the immune system, resulting in immunosuppression and increased susceptibility to viral infection [12]. In the case of HCW, an already high-risk population, this greater susceptibility to infection may lead to an unnecessary reduction in our healthcare workforce. In addition, higher GAD-7 scores have been found to correlate with functional impairment, in measures such as work productivity and health care utilization [13]. In the setting of a workforce already burdened with excessive levels of burnout, HCW will undoubtedly find it increasingly arduous to perform to their full potential while caring for critically ill patients during this healthcare crisis.

Sources of anxiety for ENT specialists appeared to centre around two key factors: increased vulnerability to infection and lack of control over infection risk. The vast majority (84\%) of participants in this study reported that they felt more likely to contract COVID-19 than their colleagues in other medical specialties. ENT specialists perform a high volume of AGPs and work in close proximity to the upper aerodigestive tract, where the greatest viral load has been detected [6]. Therefore, it is reasonable for ENT specialists to perceive themselves as being more vulnerable. In addition, one of the first documented fatalities of COVID-19 in a physician was an otolaryngologist in Wuhan, China [14]. Although evidence of the high risk status of ENT surgeons is largely anecdotal, it was well represented in this study with $11 \%(n=4)$ of participants contracting COVID-19 infection. While our data highlights increased personal risk as a key component of anxiety for ENT specialists, quantitative data can be limited in understanding the more nuanced predictors of human anxiety. A qualitative study published in JAMA utilized open questioning techniques to understand the sources of anxiety amongst health care professionals during the pandemic. They identified infecting and/or being unable to support their family if work hours increase or they develop COVID-19 as key factors to HCW anxiety [15]. One effective method of addressing ENT specialist's anxiety would be ensuring an abundant supply of PPE is available for all direct patient contacts. In this study, 58\% of participants reported that PPE was available for all patient encounters, but a considerable $40 \%$ of participants felt that PPE was only available for most encounters. The adequate provision and appropriate use of PPE is the cornerstone of hospital-based 
infection prevention and control measures and should invariably be provided to prevent COVID-19 transmission during patient contact, and provide ENT specialists with a more robust sense of control over their health [16].

In this study, one of the major mechanisms contributing to ENT specialist anxiety was a lack of control over the high-risk environment; they are currently working in. Nearly one-third (32\%) of participants reported that they felt unable to protect themselves from COVID-19 infection, suggesting the sources of infection are unpredictable and difficult to control. This is further supported by the $89 \%$ of participants who experienced a reduction in team members during the COVID-19 pandemic, most of which were due to close contacts. The reduction of the healthcare workforce may have detrimental effects on the quality of patient care, as well as HCW anxiety, via a couple of mechanisms. Delivering an effective healthcare service with a reduced workforce requires longer working hours and a higher pressure working environment, leading to physical and mental exhaustion, as well as an increased risk of medical errors [17]. In addition, a significant number of $\mathrm{HCW}$ are required to adopt less familiar or more advanced roles in order to meet the new healthcare demands. These are difficult, albeit necessary changes further increase the psychological burden on $\mathrm{HCW}$, and the chance of errors occurring in the workplace. The changing roles and reduction in our healthcare workforce will also have substantial implications for the return of routine health services following the pandemic. The resumption of elective surgeries and outpatient clinics, and subsequent increase in workload, will require a full workforce of highly skilled, ENT-trained healthcare staff to provide a high-quality ENT service. Attempting to deliver a preCOVID healthcare service with limited staff, functioning in unfamiliar roles will almost certainly result in second-rate healthcare. Additionally, expecting our remaining workforce to perform to levels exceeding their capacity will expose our remaining workforce to undue psychological distress. Therefore, we must deploy resources to understand and target potential causes of the clusters of COVID-19 outbreaks in $\mathrm{HCW}$, such as $\mathrm{HCW}$-to-HCW transmission, to protect our healthcare workforce, alleviate $\mathrm{HCW}$ anxiety and optimize our ability to provide an effective healthcare service in the post-COVID era [18].

The majority of ENT participants $(n=24,63 \%)$ felt tracheostomy formation was the highest risk procedure. Tracheostomy is a significant vector for viral transmission, both intraoperatively and during the entire post-operative course, due to the high level of nursing care they require. Additionally, mechanical ventilation in COVID-19 patients commonly requires ENT surgeons to manage their tracheostomy. Experiences from SARS in 2003 found that a single tracheostomy case could result in viral transmission to 9 HCW, despite full PPE [19]. Twenty-four percent $(n=9)$ of participants reported that managing epistaxis provided most exposure to viral transmission. Patients with epistaxis frequently present acutely to the emergency department, requiring ENT specialists to manage patients urgently in a busy and occasionally crowded environment. Moreover, the requirement to treat these patients emergently often precludes COVID-19 testing and the presence of SARS-CoV-2 in the nose, nasopharynx and blood of COVID-19 patients provides multiple potential routes for transmission $[6,20]$. Flexible nasendoscopy (FNE) was chosen by $13 \%(n=5)$ of participants as the riskiest procedure. FNE is performed in high volumes in Ireland and the UK. As for many procedures that manipulate the upper respiratory tract, FNE is potentially an AGP, with coughing, sneezing and gagging associated with droplet and aerosol formation which can infect the endoscopist [21]. The Irish National Clinical Programme in Surgery (NCPS) have provided guidance on performing FNE during the COVID-19 pandemic [22]. Additionally, innovators from across the globe have developed novel methods of reducing exposure to ENT surgeons while performing FNE during these extraordinary times.

The psychological impact of the COVID-19 pandemic can potentially compromise healthcare workers' mental health and morale, reducing workplace productivity and causing feelings of helplessness. During these times, access to psychosocial support is critical to prevent HCW burnout, depression and anxiety [23]. Twenty-two out of 38 ENT specialists were aware of the available supports from their institution, the HSE or RCSI. Increasing awareness of psychological support services available to ENT specialists and all HCW is crucial to maintaining a well-staffed workforce, and minimizing the impact the pandemic has on their mental health. Psychological support services provided include free-of-charge psychological counselling, weekly webinars and podcasts focused on mental well-being and self-care, and 24-h support helpline.

\section{Conclusion}

The COVID-19 pandemic has placed considerable strain on the mental health of ENT specialists in Ireland. Contributing factors include a feeling of increased exposure to infection compared with other medical specialties, a lack of control over contracting SARS-CoV-2 and the implications this may have for their family. Future institutional and national interventions must focus on increasing awareness of the existing psychological support services, in addition to ensuring adequate resourcing of mental health services during the COVID-19 pandemic.

Author contribution All authors contributed substantially to study design, data collection and analysis and manuscript production. All 
authors have reviewed this manuscript prior to submission and consent to it being submitted for publication

Data availability Available on request from corresponding author.

\section{Declarations}

Ethical approval Ethical approval was obtained from the National Research Ethics Committees for COVID-19-related Health Research (NREC COVID-19). Application number 20-NREC-COV-002.

Consent to participate All participants provided consent to be involved in this study.

Research involving human and animal participants This study involving human participants was carried out in accordance with the ethical standards of the institutional research committee and the 1964 Helsinki declaration and its later amendments or comparable ethical standards.

Conflict of interest The authors declare that they have no conflict of interest.

\section{References}

1. European Centre for Disease Prevention and Control (2020) COVID-19 situation update worldwide. Available at: https:// www.ecdc.europa.eu/en/geographical-distribution-2019-ncovcases2019-ncov-cases. [Accessed 9 Aug 2020]

2. COVIDSurg Collaborative (2020) Global guidance for surgical care during the COVID-19 pandemic. Br J Surg. https://doi. org/10.1002/bjs.11646

3. Health Protection Surveillance Centre (2020) Epidemiology of COVID-19 in Ireland. Available at: https://www.hpsc.ie/a-z/ respiratory/coronavirus/novelcoronavirus/casesinireland/ epide miologyofcovid-19ini reland/COVID-19_Daily_ epidemiology_report_(NPHET)\%20_website.pdf. [Accessed 10 Aug 2020]

4. McAlonan GM, Lee AM, Cheung C et al (2007) Immediate and sustained psychological impact of an emerging infectious disease outbreak on health care workers. Can J Psychiatry. https://doi. org/10.1177/070674370705200406

5. Hayes B, Prihodova L, Walsh G et al (2019) Doctors don't Dolittle: a national cross-sectional study of workplace well-being of hospital doctors in Ireland. BMJ Open. https://doi.org/10.1136/ bmjopen-2018-025433

6. Zou L, Ruan F, Huang M et al (2020) SARS-CoV-2 viral load in upper respiratory specimens of infected patients. N Engl J Med. https://doi.org/10.1056/NEJMc2001737

7. The Irish Head and Neck Society (2020) Considerations on H\&N during COVID-19. Available at: https://www.rcsi.com/dublin/ coronavirus/surgical-practice/clinical-guidance-for-surgeons. [Accessed 10 Aug 2020]

8. Spitzer RL, Kroenke K, Williams JB, Lowe B (2006) A brief measure for assessing generalized anxiety disorder: the GAD-7. Arch Intern Med. https://doi.org/10.1001/archinte.166.10.1092
9. Paiva CE, Martins BP, Paiva BS (2018) Doctor, are you healthy? A cross-sectional investigation of oncologist burnout, depression, and anxiety and an investigation of their associated factors. BMC Cancer. https://doi.org/10.1186/s12885-018-4964-7

10. Yang X, Zhang Y, Li S, Chen X (2020) Risk factors for anxiety of otolaryngology healthcare workers in Hubei province fighting coronavirus disease 2019 (COVID-19). Soc Psychiatry Psychiatr Epidemiol. https://doi.org/10.1007/s00127-020-01928-3

11. Pappa S, Ntella V, Giannakas T et al (2020) Prevalence of depression, anxiety, and insomnia among healthcare workers during the COVID-19 pandemic: a systematic review and meta-analysis. Brain Behav Immun. https://doi.org/10.1016/j. bbi.2020.05.026

12. Godbout JP, Glaser R (2006) Stress-induced immune dysregulation: implications for wound healing, infectious disease and cancer. J Neuroimmune Pharmacol. https://doi.org/10.1007/ s11481-006-9036-0

13. Ruiz MA, Zamorano E, Garcia-Campayo J et al (2011) Validity of the GAD-7 scale as an outcome measure of disability in patients with generalized anxiety disorders in primary care. J Affect Disord. https://doi.org/10.1016/j.jad.2010.07.010

14. Chen JYK, Wong EWY, Lam W (2020) Practical aspects of otolaryngologic clinical services during the 2019 novel coronavirus epidemic: an experience in Hong Kong. JAMA Otolaryngology- Head \& Neck Surgery. https://doi.org/10.1001/ jamaoto.2020.04882020.0488

15. Shanafelt T, Ripp J, Trockel M (2020) Understanding and addressing sources of anxiety among healthcare professionals during the COVID-19 pandemic. JAMA. https://doi.org/10.1001/ jama.2020.5893

16. Nguyen LH, Drew DA, Graham MS et al (2020) Risk of COVID19 among front-line health-care workers and the general community: a prospective cohort study. Lancet Public Health. https:// doi.org/10.1016/S2468-2667(20)30164-X

17. West CP, Tan AD, Habermann TM et al (2009) Association of resident fatigue and distress with perceived medical errors. JAMA. https://doi.org/10.1001/jama.2009.1389

18. Chang D, Xu H, Rebaza A et al (2020) Protecting health-care workers from subclinical coronavirus infection. Lancet Respir Med. https://doi.org/10.1016/S2213-2600(20)30066-7

19. Ofner M, Lem M, Sarwal S et al (2003) Cluster of severe acute respiratory syndrome cases among protected health care workersToronto. Can Commun Dis Rep. PMID:12794968

20. Wang W, Xu Y, Gao R et al (2020) Detection of SARS-CoV-2 in different types of clinical specimens. https://doi.org/10.1001/ jama.2020.37862020.3786

21. Meccariello G, Gallo O (2020) What ENT doctors should know about COVID-19 contagion risks. Head Neck. https://doi. org/10.1002/hed.26190

22. The National Clinical Programme in Surgery. Interim clinical guidance on flexible nasendoscopy (FNE) and nasal endoscopy in Ireland during COVID-19 pandemic. Available at https://www. rcsi.com/dublin/coronavirus/surgical-practice/clinical-guidancefor-surgeons. [Accessed 10 Aug 2020]

23. Southwick SM, Vythilingam M, Charney DS (2005) The psychobiology of depression and resilience to stress: implications for prevention and treatment. Annu Rev Clin Psychol. https://doi. org/10.1146/annurev.clinpsy.1.102803.143948 